\title{
Scintillation reduction in pseudo Multi-Gaussian Schell Model Beams in the maritime environment
}

\author{
C. Nelson ${ }^{1 *}$, S. Avramov-Zamurovic ${ }^{2}$, O. Korotkova ${ }^{3}$, S. Guth ${ }^{4}$, and R. Malek- \\ Madani $^{4}$ \\ ${ }^{I}$ Electrical and Computer Engineering Department, US Naval Academy, 105 Maryland Avenue, Annapolis, \\ MD 21402, USA \\ ${ }^{2}$ Weapons and Systems Engineering, US Naval Academy, 105 Maryland Avenue, Annapolis, MD 21402, USA \\ ${ }^{3}$ Department of Physics, University of Miami, FL, USA \\ ${ }^{4}$ Department of Mathematics, US Naval Academy, 589 McNair Rd, Stop 10M, Annapolis, MD 21402, USA \\ *cnelson@usna.edu
}

Irradiance fluctuations of a pseudo Multi-Gaussian Schell Model beam propagating in the maritime environment is explored as a function of spatial light modulator cycling rate and estimated atmospheric turnover rate. Analysis of the data demonstrates a strong negative correlation between the scintillation index of received optical intensity and cycling speed for the estimated atmospheric turnover rate.

Key words: pseudo partially coherent beam; scintillation index; maritime; multiGaussian Schell model; free-space optical communications

\section{Introduction}

Free-space optical (FSO) communication offers a natural complement and extension to current RF infrastructure and capabilities of the US Navy with the advantage of high bandwidth, low probability of detection and interception, and resistance to jamming [1,2]. FSO communications have drawbacks as well. Specifically, a propagating laser beam in the maritime environment faces challenges from optical turbulence in the atmospheric channel. One widely accepted method for potential mitigation of the optical turbulence effects on a propagating optical beam is based on modifying the spatial partial coherence of the source and use of spatially partially coherent beams (PCB) [3 - 9].

While PCBs have been the focus of much recent research, very little has been done in the area of pseudo partially coherent beams (PPCB); and what has been done has primarily focused on in-laboratory results and numerical simulations [10-15]. This paper is motivated by studies carried out in Refs. [10] and [15]. Ref. [10] first proposed and coined the term 'pseudo' in order to describe and distinguish the effect of more experimentally realistic PPCBs from the more common analytic PCBs. Specifically, the PPCB is an experimental realization of a partially coherent beam (PCB) in that the beam is physically limited in how fast individual source realizations are produced as compared with the detection rate as well as atmospheric turnover time. Ref. [15] proposed and numerically investigated the effect of the relative changing frequency of random phase screens to the atmospheric rate, or $K$ value, on scintillation index for spatially pseudo partially coherent GSM beams in atmospheric turbulence. The current paper extends the body of literature to include the experimental exploration of PPCBs through atmospheric turbulence in the maritime environment, the investigation of the effects of a varying $K$ value on the scintillation index, and the pseudo Multi-Gaussian Schell Model (MGSM) beam class. To generate the PPCBs in the experiments, we used a liquid crystal spatial light modulator (SLM) with fully controllable phase 
modulation capabilities. While the SLM is an efficient method of modulating the phase spatially, it has drawbacks associated with cycling speed limitations. Other methods to produce PPCBs include a rotating grounded glass plate $[10,11]$ and a coupled output of a superliuminescent diode to a multi-mode fiber that allows for generation of the PPCBs with a high phase fluctuation rate [12].

Analysis of the data demonstrates a strong negative correlation between the scintillation index of the received optical intensity and the SLM cycling rates for a given atmospheric turnover rate.

\section{Generation of pseudo Multi-Gaussian Schell Model Beams}

A recently developed model for the MGSM (flattop) beams, gives the following spectral (scalar) degree of coherence at the source $[16,17]$ :

$$
\mu^{(0)}\left(\rho_{1}, \rho_{2}\right)=\frac{1}{C_{0}} \sum_{m=1}^{M}\left(\begin{array}{l}
M \\
m
\end{array}\right) \frac{(-1)^{m-1}}{m} \exp \left[-\frac{\left|\rho_{2}-\rho_{1}\right|^{2}}{2 m \delta^{2}}\right]
$$

where $\rho_{1}$ and $\rho_{2}$ are position distances and superscript (0) refers to the source plane,

$$
C_{0}=\sum_{m=1}^{M}\left(\begin{array}{l}
M \\
m
\end{array}\right) \frac{(-1)^{m-1}}{m}
$$

is the normalization factor used for obtaining the same maximum intensity level for any number of terms $M$ in the summation, and $\left(\begin{array}{l}M \\ m\end{array}\right)$ is the binomial coefficient. In Eq. (1), $\delta$ is the r.m.s. width of the degree of coherence which describes the degree of coherence of the beam; where a value of $\delta=0$ gives a spatially incoherent beam and a value of $\delta \rightarrow \infty$ gives a spatially coherent beam. Additionally, the upper index $M$ relates to the flatness of the intensity profile formed in the far field: $M=1$ corresponds to the classical Gaussian Schell-Model source and $M \rightarrow \infty$ corresponds to sources producing far fields with flat centers and abrupt decays at the edges.

In our experiment, we generated the pseudo MGSM beams with phase screen realizations on an SLM with resolution of $256 \times 256$ pixels. Reference [9] describes the general process that we used for generating the SLM phase screens. To summarize the method: a 256 x 256 random matrix with Gaussian statistics and zero mean is convolved with a Multi-Gaussian window function of the form of Eq. (1), the result is then optimized for a 256 ( 8 bit) gray-scale bitmap (phase screen, see Fig. 1(a)) and sent to the SLM. Note, when these phase screens are used with an SLM, there is a zeroth order spot, which is a common artifact to the SLMs [18] and is caused by pixellation of the SLM. To eliminate the effect of the zeroth order 'hot' spot we generated "shifted" beams by multiplying the window function by a cosine function with prescribed periodicity and then convolved the result and optimized it as before. The cosine multiplication serves to generate additional spatially shifted copies of the beam, which with an experimentally prescribed periodicity closely overlap into shifted off-axis quadrants. Figure 1 illustrates the effects of representative phase screens with and without the cosine shift. Specifically, Fig. 1a shows a representative non-cosine shifted MGSM phase screen, Fig. 1b shows the graphical simulation of the stationary phase screen in Fig. 1a, and Fig. 1c shows the 'smoothed' experimental realization of Fig. 1a with a $333 \mathrm{~Hz}$ cycling rate. Fig. 1d shows a representative cosine shifted MGSM phase screen, Fig. 1e shows the graphical simulation of the phase screen in Fig. 1d, and Fig. 1f shows the experimental realization of Fig. 1d with the beam shifted into quadrants and isolation of the first order mode (upper left) with a mechanical iris. The 'cross' pattern seen on the mechanical iris (Figure 1f) is due to the 
square shape of the SLM used in the experiment. All experimental evaluations in this paper were performed using the shifted beams.

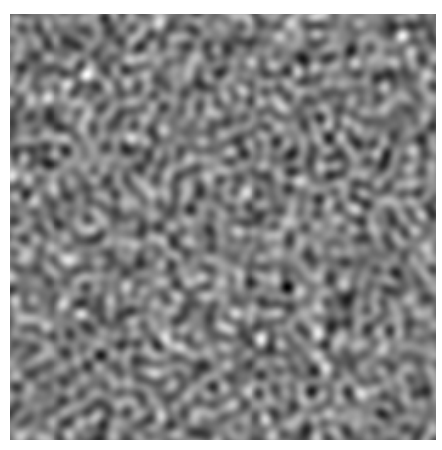

a)

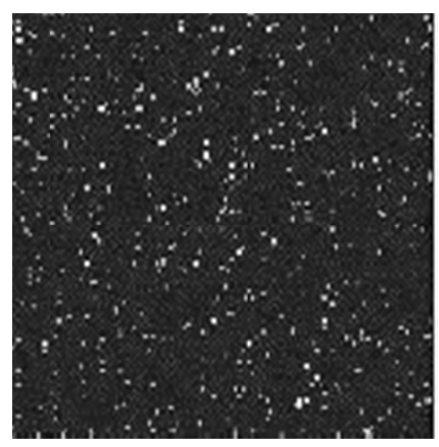

d)

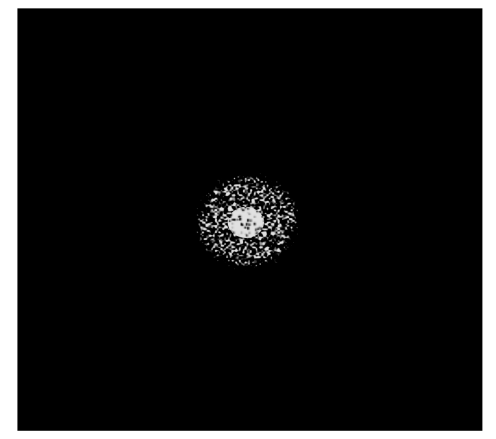

b)

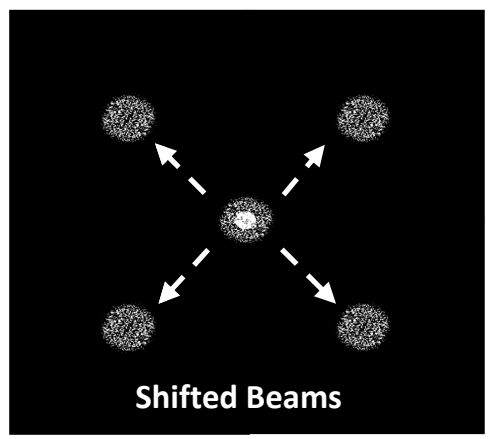

e)

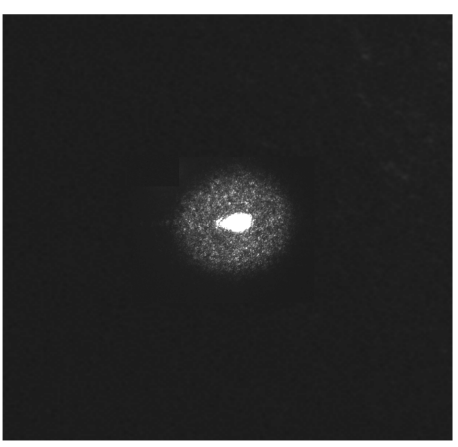

c)

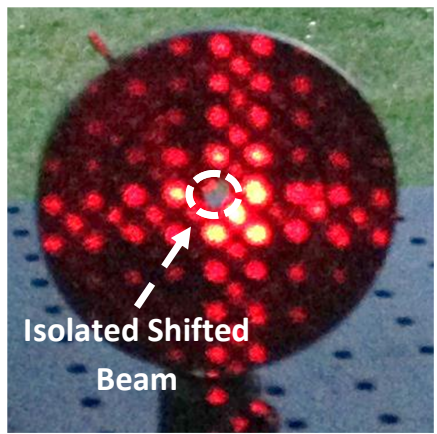

f)

Figure 1 - Typical phase screens with and without a prescribed cosine shift, simulations of the farfield, and experimental realizations: a) phase screen without cosine shift, b) simulation of phase screen in far-field, c) experimental realization with $333 \mathrm{~Hz}$ cycling, d) phase screen with cosine shift, e) simulation of far-field showing effect of the cosine shift, f) experimental realization (as used in field test).

\section{Experimental Description}

Fig. 2(a) illustrates the over-the-water (College Creek) link at the United States Naval Academy. The field trials were conducted in July and were performed during the night in relatively calm $(0$ to $1 \mathrm{~m} / \mathrm{s}$ crosswind speeds) weather conditions over a maritime link of 323 meters. A scintillometer (Fig. 2b) was used to measure the refractive index structure parameter, $C_{n}^{2}$, and anemometers were used at both the transmitter and receiver to measure the crosswind speed. Scintillometer measurements of $C_{n}^{2}$ were $3 \cdot 10^{-14} \mathrm{~m}^{-2 / 3}$, and the average cross-wind speed between the transmitter and receiver was $\sim 0.5 \mathrm{~m} / \mathrm{s}$. A low power, $2 \mathrm{~mW}$, He-Ne laser $(\lambda=632.8 \mathrm{~nm})$ was used as the light source, and a beam expander adjusted the beam size in order to fill the SLM with a reflective area of 6.14 x $6.14 \mathrm{~mm}$. For our SLM phase screen generation [19], an alternative measure of the r.m.s. phase correlation is used which gives directly the correlation term in number of pixels, $\gamma_{\varphi}$, rather than in millimeters as described in Section 2 for $\delta$. In order to relate the two parameters, $\gamma_{\varphi}$, and $\delta$, the following scaling law is used 


$$
\delta=\frac{L}{N} \times \gamma_{\varphi},
$$

where $L$ stands for the linear dimension of the (square-shaped) SLM and $N$ stands for the number of pixels in one row. For example, for the case of $\gamma_{\varphi}^{2}=128$ pixels $^{2}$, the approximate r.m.s phase correlation size, $\delta$, is computed as follows: the SLM array has $256 \times 256$ pixels over $6.14 \mathrm{~mm}$ by $6.14 \mathrm{~mm}$ and thus $\delta=\frac{6.14 \mathrm{~mm}}{256} \times \sqrt{128}=0.27 \mathrm{~mm}$. The MGSM beams for the field trials in this paper had a $\delta$ value of 0.27 $\mathrm{mm}$ and a mechanical iris (Fig. 2b) was used to isolate the shifted MGSM from the rest of the diffraction modes.

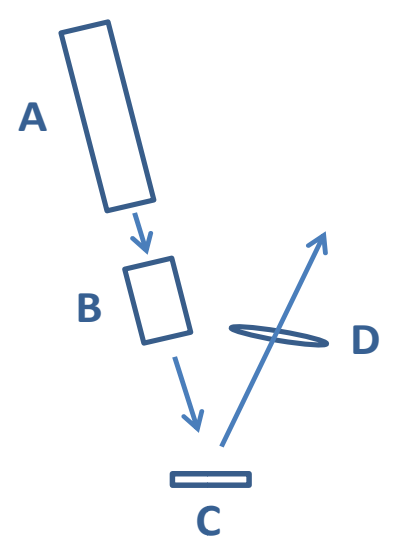

a)

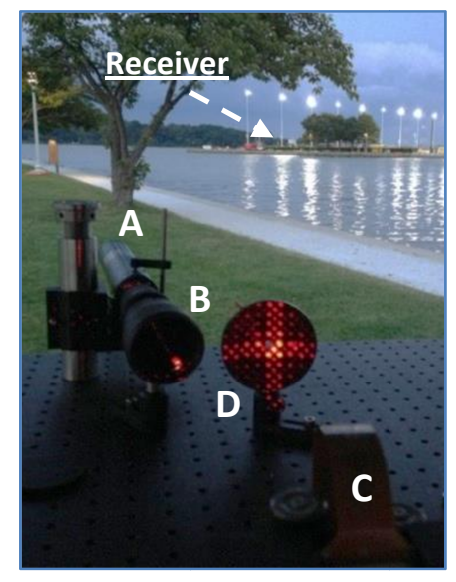

b)

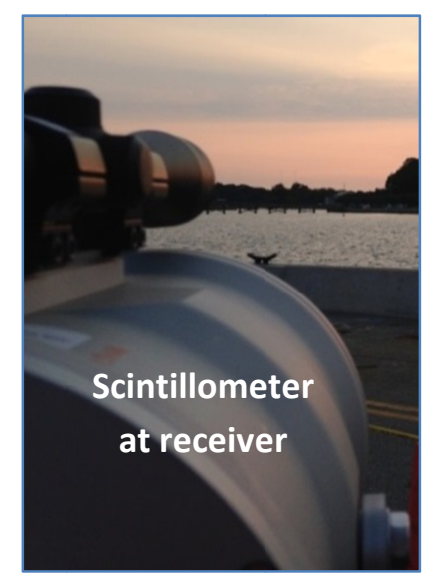

c)

Figure 2 - experimental set-up: a) transmitter side including laser (A), collimator (B), spatial light modulator (C), and mechanical iris (D), b) photo of experimental set-up, c) scintillometer at the receiver.

At the receiver, a CCD camera was used with a recording rate of 10 frames per second, 8 bit amplitude sensitivity, a sensor of $7.62 \times 7.62 \mathrm{~mm}$ in size with resolution of $1024 \times 1280$ pixels, giving an area of $4.65 \mu \mathrm{m}^{2}$ per pixel and 1.3 million intensity readings. The pixels were binned in software to create effective grids of $20 \times 20$ pixels in order to enhance the intensity resolution. Additionally, we used a red bandpass filter to minimize background lighting and measured background levels which were subsequently subtracted from the data collected to remove the bias. The scintillation index was computed from the following equation [20]

$$
\sigma_{I}^{2}=\frac{\left\langle I^{2}\right\rangle-\langle I\rangle^{2}}{\langle I\rangle^{2}}
$$

where $I$ represents the measured camera intensity.

In order to explore the effects of a pseudo MGSM beam in the maritime environment we used the relative modulation frequency, or $K$ value, which gives the ratio of the screen cycling rate to the atmospheric turnover rate [15]. As optical scintillations are caused primarily by atmospheric irregularties with a size on the order of the First Fresnel Zone (FFZ) we estimated the atmospheric turnover rate by dividing the average crosswind speed between the transmitter and the receiver by the FFZ [21]. The FFZ is defined by $\sim(\lambda D)^{1 / 2}$, where $\lambda$, is the wavelength and $D$ is length of the propagation channel. For our 
case, with $\lambda=632.8 \mathrm{~nm}$, and $D=323 \mathrm{~m}$ the FFZ was $\sim 1.43 \mathrm{~cm}$ and with an average crosswind speed of $\sim 0.5 \mathrm{~m} / \mathrm{s}$ the approximate atmospheric turnover rate was $\sim 35 \mathrm{~Hz}$. Due to our limited SLM cycling rate, one of our goals was to conduct the experimentation on a relatively calm evening in order to achieve large $K$ values. For each of four data points, with approximate $K$ values of 2.9, 5.7, 7.1, and 9.5 (corresponding to the SLM cycling rates of 100,200, 250, and $333 \mathrm{~Hz}$ respectively) three consecutive one-minute runs were performed to assure data repeatability. Note, for a pure PCB the $K$ value would be infinite which is not necessarily practical for modern systems except possibly for Efimov et al [12] where a coupled output of a superliuminescent diode to a multi-mode fiber was employed to produce PCBs with a high phase fluctuation rate.

\section{Results}

Figure 3 shows the results of scintillation index vs. cycling rate for the three trial runs. The trend is apparent even with the 1.96 sigma error bars.

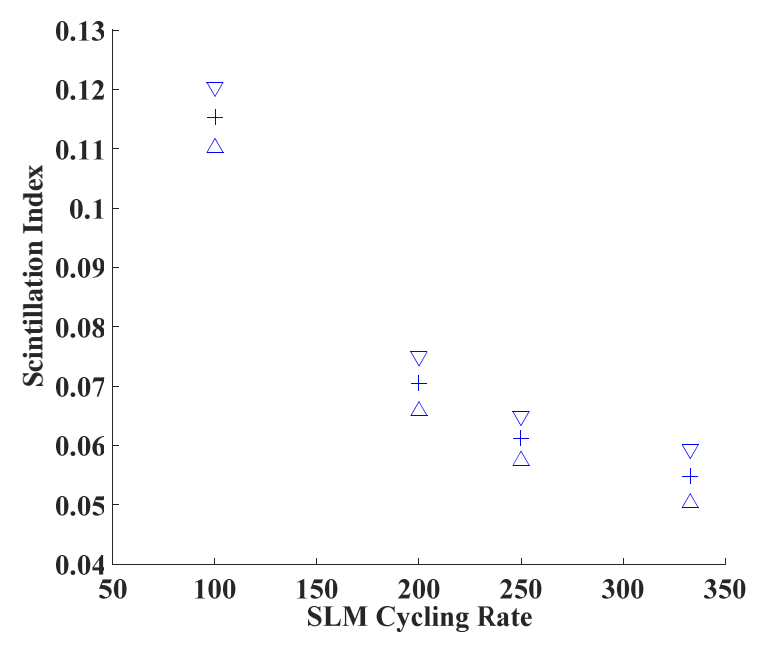

a)

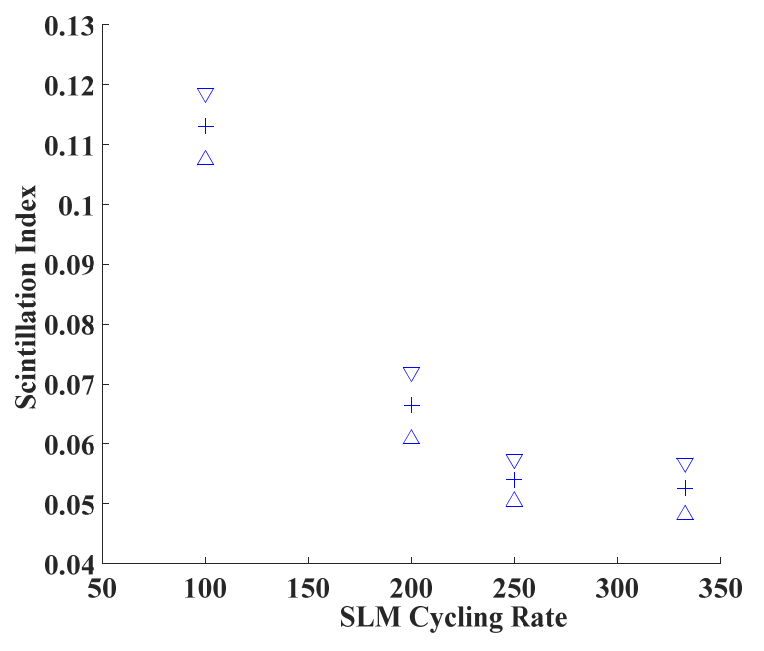

b)

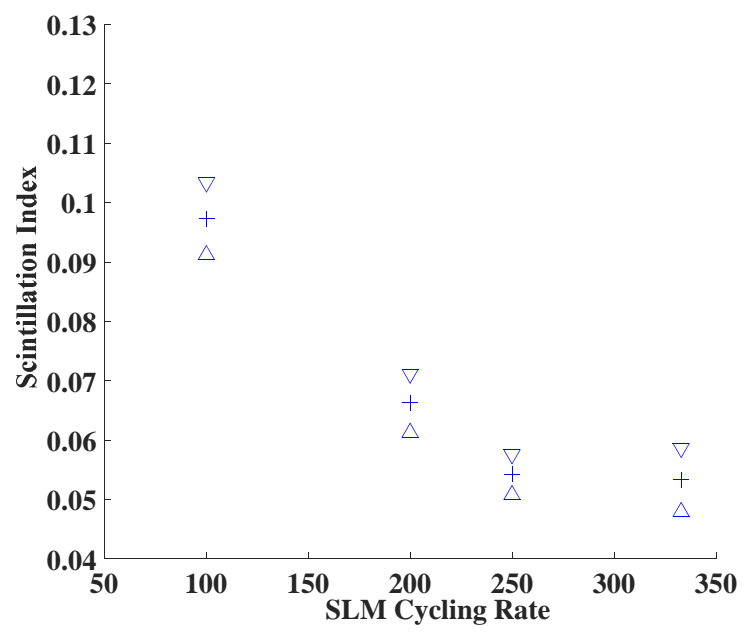

c) 
Figure 3 - Scintillation index vs. phase screen cycling rate MGSM beam, $C_{n}{ }^{2} \sim 3 \cdot 10^{-14} \mathrm{~m}^{-2 / 3}$, a cross wind speed average of $\sim 0.5 \mathrm{~m} / \mathrm{s}, K$ values of $\sim 2.9,5.7,7.1$, and 9.5 respectively for cycling rates of 100, 200, 250, and $333 \mathrm{~Hz}$ : a) trial one, b) trial two, c) trial three. Error bars are at 1.96 sigma.

Figure 4 gives a representative example of the data collected. Fig. 4(a) shows the scatterplot of $20 \times 20$ average binned camera pixel intensity values over one minute as compared with the scintillation index as computed from Eq. 3. Fig. 4(b) shows the time series of 20x20 binned camera intensity values over the one-minute data capture. The scatterplot shows the lack of correlation between the camera intensity and scintillation index, which is expected, and the time series gives a general representation of the random fluctuations of intensity during a one-minute run of laser beam propagation in the maritime environment.

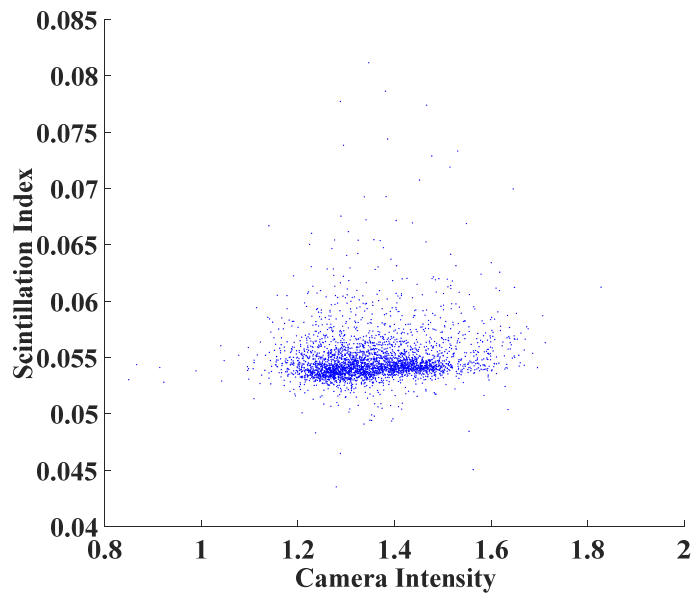

a)

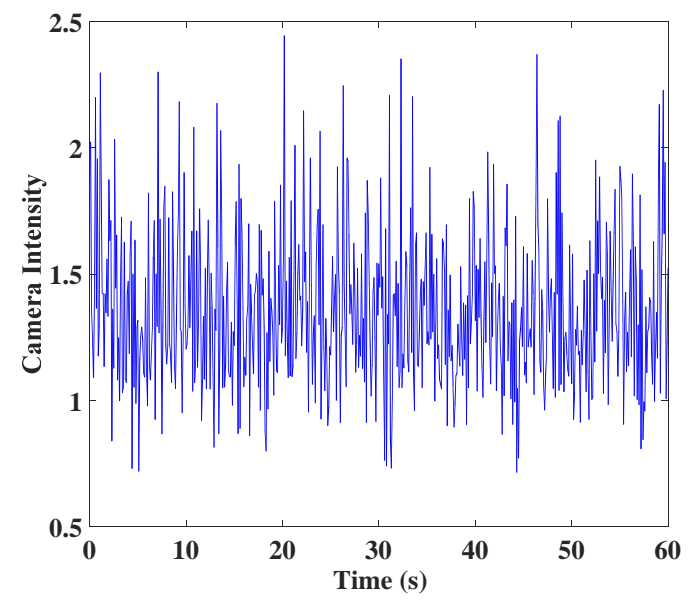

b)

Figure 4 - Representative plots of average binned camera pixel intensities ( $333 \mathrm{~Hz}$ phase screen cycling rate): a) scintillation index vs. intensity, and b) time series of intensity measured seconds.

Table 1 summarizes the results from the experiments. Specifically, each trial is illustrated with the average scintillation index for each $K$ value. The percent reduction of scintillation index from a $K$ value of $\sim 2.9$ to a $K$ value of $\sim 9.5$ was given as a comparison value to the simulations in Ref. [15].

\begin{tabular}{|c|c|c|c|c|c|}
\hline Trial & $\begin{array}{c}\boldsymbol{\sigma}_{\boldsymbol{I}}^{\mathbf{2}} \\
(\boldsymbol{K} \sim \mathbf{2 . 9})\end{array}$ & $\begin{array}{c}\boldsymbol{\sigma}_{\boldsymbol{I}}^{\mathbf{2}} \\
(\boldsymbol{K} \sim \mathbf{5 . 7})\end{array}$ & $\begin{array}{c}\boldsymbol{\sigma}_{\boldsymbol{I}}^{\mathbf{2}} \\
(\boldsymbol{K} \sim \mathbf{7 . 1})\end{array}$ & $\begin{array}{c}\boldsymbol{\sigma}_{\boldsymbol{I}}^{\mathbf{2}} \\
(\boldsymbol{K} \sim \mathbf{9 . 5})\end{array}$ & $\begin{array}{c}\text { \% Reduction in } \boldsymbol{\sigma}_{\boldsymbol{I}}^{\mathbf{2}} \\
\text { From } \boldsymbol{K} \sim \mathbf{2 . 9} \text { to } \boldsymbol{K} \sim \mathbf{9 . 5})\end{array}$ \\
\hline $\mathbf{1}$ & 0.115 & 0.070 & 0.061 & 0.055 & 52.2 \\
\hline $\mathbf{2}$ & 0.113 & 0.066 & 0.054 & 0.053 & 53.1 \\
\hline $\mathbf{3}$ & 0.097 & 0.066 & 0.054 & 0.053 & 45.4 \\
\hline
\end{tabular}

Table 1 - Scintillation index values as a function of cycling rate for three trials

These results show a $50.2 \%$ average scintillation index reduction going from a $K$ value of $\sim 2.9$ to a $K$ value of $\sim 9.5$, which compares favorably with the results from Qian's simulations which showed an approximately $50 \%$ reduction for a PPCB going from a $K$ value of 3 to 10 [15]. Additionally, these results are consistent with findings of PPCB beam propagation over 1 meter utilizing an in-laboratory hotair turbulence emulator [22]. 


\section{Conclusions}

We have successfully demonstrated a maritime propagation link with a pseudo Multi-Gaussian Schell Model beam and have shown the agreement of scintillation index with published simulation results. Analysis of the data demonstrates a strong negative correlation between the scintillation index of received optical intensity and cycling rates for a given atmospheric turnover rate. Our results are of importance for practical implementation of source partial coherence in FSO communication systems operating over maritime atmospheric channels in order to enhance their capabilities.

\section{Acknowledgements}

C. Nelson's work is supported by grant: US Office of Naval Research (ONR) grant N001614WX30023.

S. Avramov-Zamurovic and S. Guth are supported by US ONR grant N0001414-WX-00267. O. Korotkova is supported by the Air Force Office of Scientific Research (AFOSR) grant FA9550-12-10449; and the ONR grant N00014-15-1-2350. R. Malek-Madani is supported by US ONR grant N0001414-WX-00197.

\section{References}

[1] S. Das, H. Henniger, B. Epple, C. I. Moore, W. Rabinovich, R. Sova, and D. Young, "Requirements and Challenges for Tactical Free-Space Lasercomm," Milcom, (2008)

[2] J.C. Juarez, J. E. Sluz, C. Nelson, M. B. Airola, M. J. Fitch, D. W. Young, D. Terry, F. M. Davidson, J. R. Rottier, and R. M. Sova, "Free-space optical channel characterization in the maritime environment," Proc. SPIE 7685, (2010)

[3] J. C. Ricklin and F. M. Davidson, "Atmospheric optical communication with a Gaussian Schell beam," J. Opt. Soc. Am. A, vol. 20, no. 5, 2003

[4] V. L. Banach, V. A., Buldakov, V. M., Mironov, "Intensity fluctuations of a partially coherent light beam in a turbulent atmosphere," Opt. Spectrosc., vol. 54, pp. 1054-1059, 1983

[5] J. C. Ricklin and F. M. Davidson, "Atmospheric turbulence effects on a partially coherent Gaussian beam : implications for free-space laser communication," J. Opt. Soc. Am. A, vol. 19, no. 9, 2002

[6] O. Korotkova, L. C. Andrews, R. L., Phillips, "Model for a partially coherent Gaussian beam in atmospheric turbulence with application in Lasercom," Opt. Eng., vol. 43, no. 2, 2004

[7] O. Korotkova, L. C. Andrews, and R. L. Phillips, "The Effect of Partially Coherent QuasiMonochromatic Gaussian-Beam on the Probability of Fade," Proc. SPIE 5160, 2004

[8] Leader, J. C. "Intensity fluctuations resulting from partially coherent light propagating through atmospheric turbulence," J. Opt. Soc. Am. 69, 73-83 (1979).

[9] Avramov-Zamurovic, S., Korotkova, O., Nelson C., and Malek-Madani, R., "The dependence of the intensity PDF of a random beam propagating in the maritime atmosphere on source coherence," Waves in Complex and Random media 24, 69-82 (2014). 
[10] D. Voelz, and K. Fitzhenry, "Pseudo-partially coherent beam for free-space laser communication," Proc. of SPIE 5550, 218-224 (2004).

[11] G. P. Berman, A. R. Bishop, B. M. Chernobrod, O. O. Chumak, V. N. Gorshkov, S. V. Torous, "The effects of phase diffuser on scintillations of laser radiation for long-distance propagation in the atmosphere," Proc. of SPIE Vol. 7588 (2010)

[12] A. Efimov, K. Velizhanin, and G. Gelikonov, "Simultaneous scintillation measurements of coherent and partially coherent beams in an open atmosphere experiment," Proc. SPIE 8971 (2014).

[13] K. Drexler, M. Roggemann, D. Voelz, "Use of a partially coherent transmitter beam to improve the statistics of received power in a free-space optical communication system: theory and experimental results," Opt. Eng., vol. 50, no. 2, 2011.

[14] X. Xiao, and D. Voelz, "Wave optics simulation of pseudo-partially coherent beam propagation through turbulence: application to laser communications," Proc. SPIE 6404, (2006).

[15] X. Qian, W. Zhu, and R. Rao, "Numerical investigation on propagation effects of pseudo-partially coherent Gaussian Schell-model beams in atmospheric turbulence," Optics Express Vol. 17, No. 5 (2009).

[16] Sahin, S. and Korotkova, O., "Light sources generating far fields with tunable flat profiles," Opt. Lett. 37, 2970-2972 (2012).

[17] Korotkova, O., Sahin, S. and Shchepakina, E., "Multi-Gaussian Schell-model beams," J. Opt. Soc. Am. A 29, 2159- 2164 (2012).

[18] D. McGloin, G. C. Spalding, H. Melville, W. Sibbett, and K. Dholakia, “Applications of spatial light modulators in atom optics," Optics Express 11, 158-166 (2003).

[19] O. Korotkova, S. Avramov-Zamurovic, C. Nelson, R. Malek-Madani, Y. Gu, G. Gbur, "Scintillation reduction in multi-Gaussian Schell-model beams propagating in atmospheric turbulence," Proc. SPIE 9224 (2014).

[20] L. C. Andrews and R. L. Phillips, Laser Beam Propagation through Random Media, 2nd ed. Bellingham, WA: SPIE Press, 2005

[21] S. F. Lawrence, R. S., Ochs, G. R., Clifford, "Measurements of Atmospheric Turbulence Relevant to Optical Propagation," J. Opt. Soc. Am., vol. 60, no. 6, 1970.

[22] M. Lipp, "Minimizing Scintillation using Pseudo-Partially Coherent Beams," presentation at the Sixteenth Annual Directed Energy Symposium, March 2014, Huntsville, Alabama. 